\title{
Article \\ LW-FIRE: A Lightweight Wildfire Image Classification with a Deep Convolutional Neural Network
}

\author{
Amila Akagic*(D) and Emir Buza \\ Faculty of Electrical Engineering, University of Sarajevo, 71000 Sarajevo, Bosnia and Herzegovina; \\ ebuza@etf.unsa.ba \\ * Correspondence: aakagic@etf.unsa.ba
}

check for

updates

Citation: Akagic, A.; Buza, E.; LW-FIRE: A Lightweight Wildfire Image Classification with a Deep Convolutional Neural Network. Appl. Sci. 2022, 12, 2646. https://doi.org/ 10.3390/app12052646

Academic Editors: Antonio Fernández-Caballero, Byung-Gyu Kim and Hugo Pedro Proença

Received: 10 February 2022 Accepted: 26 February 2022 Published: 4 March 2022

Publisher's Note: MDPI stays neutral with regard to jurisdictional claims in published maps and institutional affiliations.

Copyright: (C) 2022 by the authors. Licensee MDPI, Basel, Switzerland. This article is an open access article distributed under the terms and conditions of the Creative Commons Attribution (CC BY) license (https:// creativecommons.org/licenses/by/ $4.0 /)$

Abstract: Analysis of reports published by the leading national centers for monitoring wildfires and other emergencies revealed that the devastation caused by wildfires has increased by 2.96 -fold when compared to a decade earlier. The reports show that the total number of wildfires is declining; however, their impact on the wildlife appears to be more devastating. In recent years, deep neural network models have demonstrated state-of-the-art accuracy on many computer vision tasks. In this paper, we describe the design and implementation of a lightweight wildfire image classification model (LW-FIRE) based on convolutional neural networks. We explore different ways of using the existing dataset to efficiently train a deep convolutional neural network. We also propose a new method for dataset transformation to increase the number of samples in the dataset and improve the accuracy and generalization of the deep learning model. Experimental results show that the proposed model outperforms the state-of-the-art methods, and is suitable for real-time classification of wildfire images.

Keywords: artificial neural networks; computer vision; machine learning; deep learning; deep neural networks; image classification; convolutional neural networks

\section{Introduction}

The recent reports from the National Interagency Fire Center (NIFC) show that the number of wildfires is decreasing on an annual level in the USA [1-3]. At the same time, the impact and devastation caused by wildfires are becoming greater as evidenced by the increasing average number of acres burned by a single wildfire. The total number of wildfires and the total number of acres burned annually over the period of the last 12 years, including the data which are partially available for the year 2021, are shown in Figure 1. In the 2020 report, 58,950 wildfires were recorded, along with 10.12 million acres of land burned as a consequence of wildfires. A decade earlier, 71,970 wildfires were recorded along with 3.42 million acres of land burned. This data shows an alarming increase in the total burned land area (2.96-fold increase), and it also shows that the overall level of devastation caused by a single wildfire has increased. On average, a single wildfire in 2020 burned 3.61 times more land than in 2010. From 2010 to 2020, on average, 63,605 wildfires per year were recorded, while 7.1 million acres per year were burned (the size of Maryland or Hawaii).

The average number of acres burned per wildfire data is presented in Figure 2. It is evident that there is an increase in the devastation caused by wildfires over the last decade. Similar trends can be observed in other places around the world. For example, in 2019, the number of wildfires in the Amazon rainforest increased by $80 \%$ compared to a year before. Approximately 75,000 wildfires were recorded just in 2019 in the Amazon rainforest. All data indicate that the development of accurate and reliable early wildfire detection systems is becoming a necessity. 

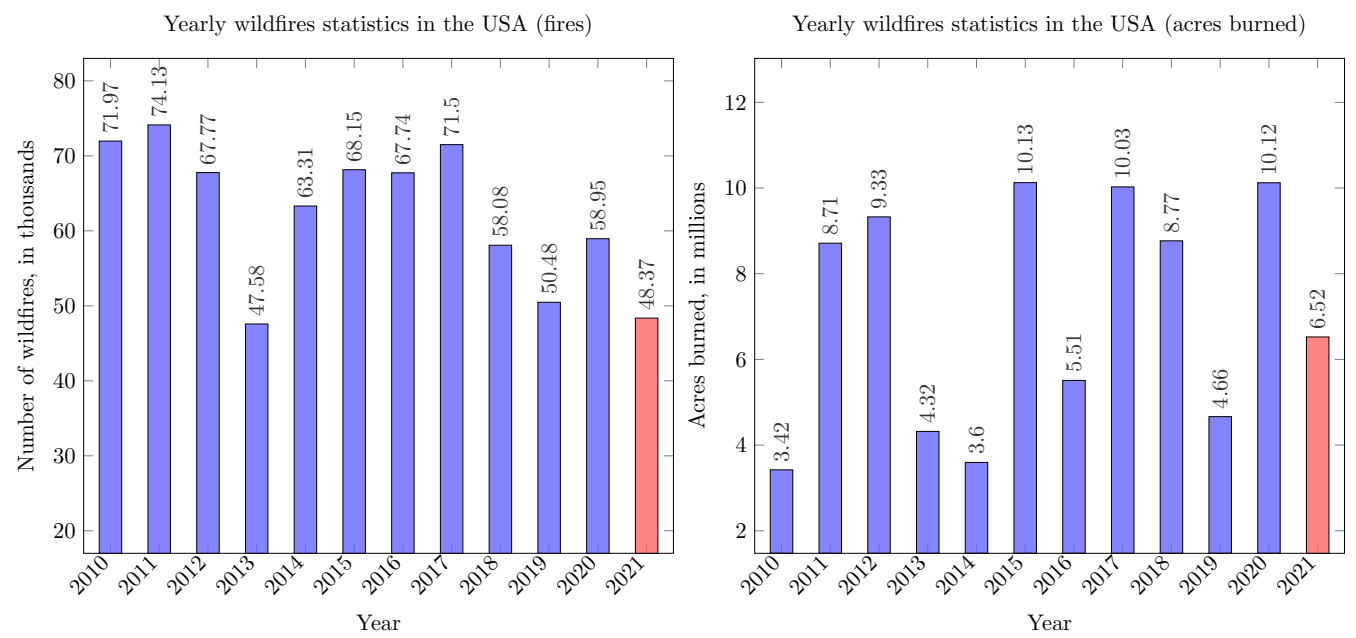

Figure 1. The number of wildfires (left) and acres burned (right) in the USA from 2010 to 2021. The information is collected from the interagency Fire and Aviation Management Web Applications (FAMWEB) system. The data for 2021 are from the period January 1 to 29 October 2021 (marked in red). Data for the last two months of the year 2021 are not available yet.

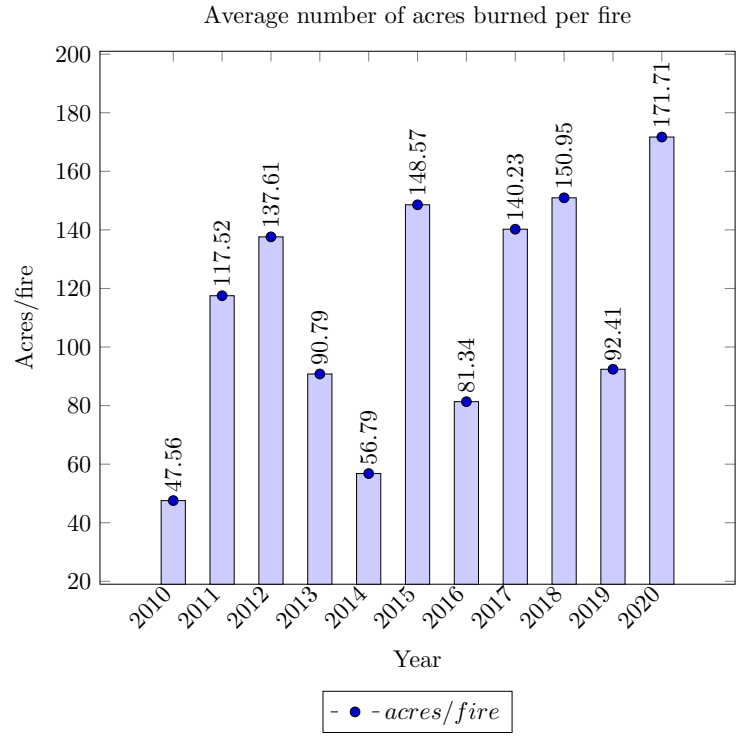

Figure 2. Average number of acres burned by wildfires from 2010 to 2020 . The number of fires decreased, but the effect of those fires is more devastating-more acres are burned by fewer fires.

Furthermore, there is also a growing awareness of the economic and social benefits associated with forests [4]. The methods for automatic wildfire detection, diagnosis, and prevention have been gaining more and more attention over the years. At the same time, remote sensing technologies and geographic information systems, as well as processes for data acquisition, are more accessible and versatile [5]. The main research challenges are associated with adequately analyzing and extracting knowledge from various sources of data. Continuous improvements in the field of artificial intelligence (AI), especially machine learning (ML), provide ample opportunities to design algorithms and methods to solve domain-specific problems, including the aforementioned problem of extracting knowledge from image data.

In recent years, computer vision-based wildfire prediction and detection systems have become very popular due to the rapid emergence and growth of technologies capable of analyzing large amounts of digital images with different artificial intelligence methods. Machine learning and deep learning (DL) methods are being increasingly applied to wildfire prediction and detection [6-9]. Unmanned aerial vehicles (UAVs) equipped with modern 
computer vision-based systems have great potential for detecting, monitoring, and fighting wildfires. A system for the automatic detection of wildfires is recommended for any wildlife preservation and protection organization, forestry management group, wildfire research organization, and other government or non-government agency.

In this paper, we propose a new method for the automated classification of wildfire images based on a modern deep learning approach for image classification. The proposed method is evaluated on two publicly available image datasets. In addition, a novel technique is presented for the efficient training of a lightweight CNN model in instances when the training data is scarce.

In this paper, a number of important contributions are made:

- A new dataset transformation method is proposed. This method increases the number of dataset samples and improves the training and generalization of the deep learning model. In instances when samples associated with one class are more prevalent than samples associated with other class(es), the proposed image dataset transformation method is able to improve the balance of the dataset;

- A new method for automatic classification of wildfire images based on a modern deep learning approach for image classification is presented. We propose a new lightweight deep convolutional neural network (CNN) model for the binary classification of wildfire images. In this paper, a certain number of lightweight configurations are considered. The results of prior empirical research, where many more configurations have been considered, indicate that the configurations that are presented in this paper offer higher performance with respect to the accuracy, execution time, and memory usage of the model. The model was tested on an additional dataset to show its capacity to generalize beyond the data it was trained on. Additionally, we extend our solution to work on multiclass classification;

- A best-performing model is identified as RT-LW-FIRE100 12 . It exhibits a range of favorable characteristics. The model is simple and can be rapidly and efficiently trained. It is capable of predicting an arbitrary image in $32 \mathrm{~ms}$, which is approximately 31 frames per second (FPS); thus, it is suitable for real-time classification of wildfire images. The model is compact and can be implemented on less demanding hardware and deployed on a lightweight UAV platform. It exhibits high accuracy on the testing subset. One of the main motivations for this work is to create a model that can be implemented on a drone to efficiently detect, monitor, and fight against situations related to wildfire outbreaks.

The remainder of this article is organized as follows. Section 2 surveys the latest developments in the wildfire classification and detection that were gathered and distilled from the literature survey. Section 3 details a new method for wildfire classification with a deep convolution neural network. In Section 4, experimental results and evaluation criteria are presented. In Section 5, a discussion and comparison of results with state-of-the-art are presented. Finally, in Section 6, some conclusions are provided.

\section{Related Work}

Numerous fire detection methods and algorithms have been proposed in the recent literature. In this section, we review some works related to wildfire full-frame classification.

Dunnings et al. in [6], used simplified and experimentally optimized AlexNet and InceptionV1 CNN architectures for a frame-based binary fire detection task. For each architecture, different network configurations were experimentally assessed. For the AlexNet architecture, the authors considered 6 variations by removing a combination of 1-3 different $\mathrm{CNN}$ layers, while for InceptionV1, the authors considered 8 variations by removing up to 8 inception modules from the original architecture. The reported results showed that the reduction in model complexity, which also resulted in a reduction in the number of parameters (in millions), improved the classification accuracy. The authors have identified the best-performing model for AlexNet and InceptionV1. The model based on AlexNet was named FireNet, while the model based on InceptionV1 was named InceptionV1-OnFire. 
The accuracies of the optimized models were compared against the original AlexNet, InceptionV1, and VGG-13 models. The reported results indicated that it is possible to achieve 93\% accuracy with InceptionV1-OnFire for the binary classification of fire images with $8.4 \mathrm{fps}$. The dataset used in this work is a collection of data from Chenebert et al. [10] (75,683 images), Steffens et al. [11] (20,593 images), also known as "furg-fire-dataset" [12], and material from public video sources (youtube.com: 269,426 images). The authors extracted 23,408 images from this collection to create a training subset, and 2931 images to create a testing subset.

Samarth et al. in [7], further explored this approach, i.e., the reduction in the complexity of the CNN model, and they have based their research on new architectures such as Inception, ResNet, and EfficientNet, and their combinations. They explored two detection problems: binary fire detection to detect the presence of fire in a frame and localization to detect the precise location of the fire within the frame. The authors explored InceptionV2, InceptionV3, InceptionV4, ResNet, a hybrid Inception-ResNet which is a combination between Inception and ResNet architectures, and EfficientNet. The authors identified the best variations, which they named as InceptionV3-OnFire and InceptionV4-OnFire. For the full-frame binary fire detection, these methods achieved $94.4 \%$ accuracy with InceptionV3-OnFire, and 95.6\% accuracy with InceptionV4-OnFire, with $13.8 \mathrm{fps}$, and $12 \mathrm{fps}$, respectively.

In [8], Thomson et al. explored two CNN architectures to improve classification performance for real-time fire detection, which was based on experiments on NasNet-AMobile [13] and ShuffleNetV2 architectures [14]. Architectures were chosen because of their compactness and ability to provide high performance on ImageNet classification. The authors used transfer learning technique, whereby some layers within the original network are frozen, some layers are removed, and new layers to be trained on new data for fire classification are added. To be more precise, they removed only the last fully connected layer and added a new linear layer for binary classification. For the first half of training time, the entire architecture was frozen except the final linear layer, whilst for the remaining half of training time, the final convolutional layer in ShuffleNetV2 was unfrozen, and the final two reductions, normal cell iterations for NasNet-A-Mobile, were also unfrozen. The dataset used for training is the same dataset used in [6]. The best models were named NasNet-A-OnFire and ShuffleNetV2-OnFire with respect to the original models. The results indicated comparable performance to the models presented in [7], where NasNet-A-OnFire achieved 95.3\% accuracy with $7 \mathrm{fps}$, and ShuffleNetV2-OnFire achieved 95\% accuracy with $40 \mathrm{fps}$.

Sousaa et al. [9] proposed a transfer learning technique to optimize the InceptionV3 model that was pre-trained on the ImageNet dataset (1000 classes). InceptionV3 is a convolutional neural network with 48 layers in total. Different types of layers are used in the model: convolutional, two types of pooling layers (max and average), fully connected, dropout, and a softmax classifier. A dataset called the cross-validation database (CVDB) was created as a subset from the Portuguese Firefighters Portal Database (PFPDB), which was created from 3900 images and images from the data augmentation. Data augmentation was used to generate more training samples and reduce overfitting. A total of 3623 fire and 3551 not fire images were used. The dataset was partitioned with k-fold cross-validation, where $\mathrm{k}=10$. The model resulted in $93.6 \% \pm 1.57$ accuracy.

Jadon et al. in [15], presented FireNet as a lightweight shallow neural network that could be employed on embedded platforms such as Raspberry Pi. The network was built from scratch and trained on a dataset that was also presented in the paper. The dataset consists of 46 fire videos (19,094 images) with 16 non-fire videos (6747 images), and an additional 160 non-fire images [16]. The model achieved 93.91\% accuracy. 


\section{Materials and Methods}

The overall design of our method is illustrated in Figure 3. We first describe the process of dataset selection. Then, we present the three major phases in the following subsections: pre-processing, a lightweight CNN for wildfire image classification, and inference.

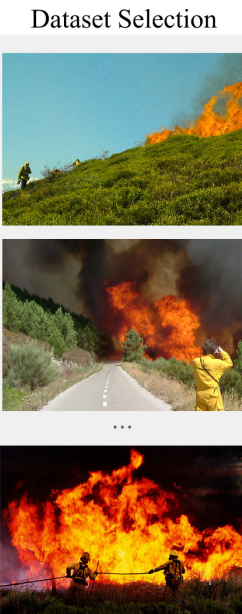

Described in Section 3.1

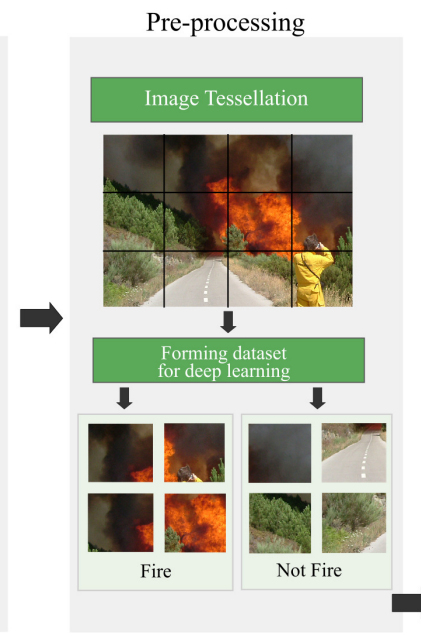

Described in Section 3.2

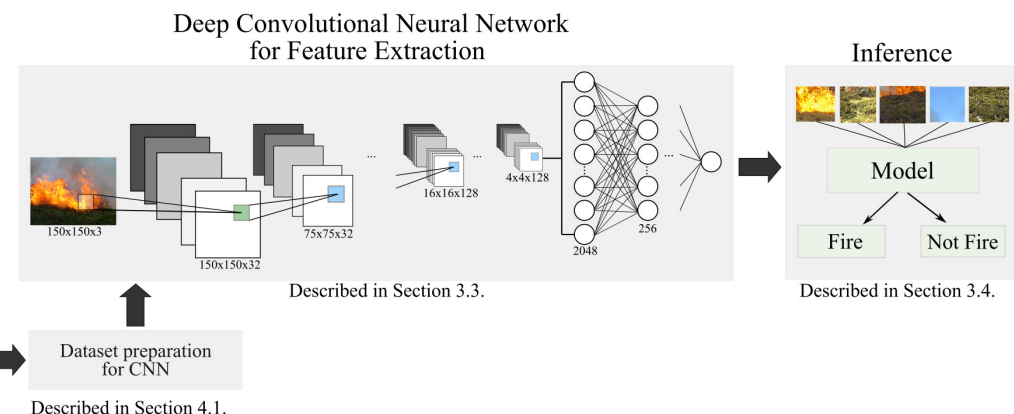

Described in Section 4.1 .

Figure 3. Overview of phases in the proposed method. We propose a new pre-processing approach to create a new dataset that consists of two classes (fire and not-fire). Then, we prepare the dataset for training, validation, and testing for the newly proposed deep convolutional neural network. The dataset is fed into the proposed lightweight CNN model to carry out binary classification.

\subsection{Dataset Selection}

From the literature review, we observed that existing datasets are mostly created from video data of fire and non-fire scenarios. The problem with this approach is that these images are very similar; the background is the same in the frames of one video, hence, the change between frames is minimal. Video sources might not be suitable for a robust and versatile deep learning model because frames are very similar, especially if the model is planned to be used in real-life wildfire scenarios.

In this work, we use the Corsican Fire DataBase (CFDB) [17,18]. This is an open image database that contains 500 visual range fire images. Image acquisitions were based on different camera positioning, vision, weather, vegetation, distance from the fire, and brightness. Every image in the dataset is characterized by 22 parameters, i.e., camera model, sensitivity, exposure time, GPS position, the direction of propagation of the fire, the color of the smoke, presence of clouds, etc. All images have been associated with the corresponding ground truth images.

In the context of the development of deep learning models and applications, this dataset is challenging, as it only contains 500 images. However, images contain reallife scenarios of wildfire images, and as such, the authors believe they can facilitate the development of a model to be applied in real-life applications. A few examples of images from this dataset are shown in Figure 3. In this paper, we show that it is possible to use the CFDB dataset to train a deep learning model.

\subsection{Pre-Processing}

In this subsection, we address the problem of having a dataset of images with a relatively small number of images. We begin by acknowledging the fact that, in order to increase the capacity of a machine learning model to generalize beyond the training data, the training dataset should have a sufficient number of statistically relevant samples.

One of the most popular techniques for increasing the size of the labeled training sets is data augmentation. In this technique, the training data is increased by applying various class-preserving transformations. This means that all subsequently augmented sub-images 
inherit the same label as the original image. This is contrary to the idea presented in this paper. The motivation for our method comes from the fact that the vast majority of CNN models resize the original image to a tensor of a specific dimension defined by a neural model, which results in the loss of valuable data. We observe that the data can be used more efficiently by representing smaller parts of the image as the input data to the model. In this process, it is important to differentiate between the data with different information, e.g., "there is a fire on the image" or "there is no fire on the image". Hence, we design a strategy that pre-processes data and then use it to obtain maximal data usage in the training process.

Since the chosen dataset contains a small number of images with favorable characteristics, in this subsection, we propose a method to increase the number of dataset samples. The proposed method can also be used to address the problem of having an imbalanced dataset, whereby images associated with one class are more frequent than the images associated with other class(es). In this paper, the proposed method for image dataset transformation is used to improve the training and generalization of the proposed model for wildfire image classifications.

The CFDB dataset contains $T=500$ images. Images are not presented with uniform resolution. The resolution of images ranges from $183 \times 242$ to $4000 \times 3000$, while the number of pixels ranges from a minimum of $44.3 \mathrm{k}$ to a maximum of $12 \mathrm{M}$ pixels. Some images contain large fire regions; however, the majority of the images contain only some small fire regions. In real-life situations, it is important to detect early signs of fire, so increasing the chance of detection of early signs of fire, which might be presented by a small region of fire in an image, is very important. Since this dataset represents important data captured in real-life scenarios, we use this dataset to create a new dataset by applying a tessellation method that is performed on original images. This results in creating many smaller images that are denoted as sub-images. Sub-images are used to increase the detection accuracy of a deep learning model, hence providing a greater possibility for the detection of wildfire in real-life scenarios.

In this phase, the images are prepared for the training process of a new lightweight CNN for wildfire image classification described in Section 3.3. The major pre-processing steps can be grouped into two stages: (1) image tessellation, and (2) formation of a dataset for deep learning.

\subsubsection{Image Tessellation}

The principal idea behind this algorithm is to mitigate the problem of information loss due to a common practice of deep learning models to resize an input image to a tensor of a specific dimension. A dimension is usually defined by a neural model that is being used. For example, VGG, InceptionNet, ResNet, and many other models resize an image into a $224 \times 224 \times 3$ tensor before the training and testing process begins. Resizing an image implies changing its dimension, which can be accomplished by changing only the width or the height, or both at the same time. The preferred method for resizing is resampling using pixel area relation. If a dataset consists of images of different resolutions, this means that all images will be resized into $224 \times 224 \times 3$. This inevitably leads to information loss, and it was one of our motivations for our work. To prevent unnecessary information loss, we propose a new method based on an image tessellation scheme to produce sub-images of comparable sizes. This method will also increase the number of dataset samples. To the best of our knowledge, this is the first time that this method was used for training a deep learning network.

Every image $I$ in the dataset is divided into an even number of sub-images, denoted as $N$. The total number of sub-images, $T_{N}$, depends only on a selected number $N$. It is independent of the image size. Image $I$ is divided into $X \times Y$ sub-images, where $X$ and $Y$ are chosen to preserve the approximate proportion of each sub-image. Since $N$ is an even number, at least one number, $X$ or $Y$, has to be an even number. Two image tessellation scenarios are considered in this paper, as shown in Table 1. Other scenarios are 
possible, but the CNN models developed under the considered scenarios exhibit satisfactory accuracy. In the first sub-stage, every image $I$ from the dataset is divided into an even number of sub-images $N$.

Table 1. Two values of $N$ divide an image from the CFDB dataset into different set of tiles, which results in different numbers of sub-images per $N$.

\begin{tabular}{ccc}
\hline $\mathbf{N}$ & Tiles $(X \times Y)$ & No. Sub-Images $\left(T_{N}\right)$ \\
\hline 12 & $4 \times 3$ & 6000 \\
\hline 42 & $7 \times 6$ & 21,000 \\
\hline
\end{tabular}

When $N=12$, the image width is divided by $X=4$, and the height is divided by $Y=3$. When $N=42$, the image width is divided by $X=7$, and the height is divided by $Y=6$. If $N=10$ or $N=40$, the image tessellation tool can result in sub-image sizes that are disproportional, e.g., $5 \times 2$ or $8 \times 5$, which should generally be avoided because images are resized before training, so data loss might occur. A better tessellation scheme can be attained with $N=42$, which results in $7 \times 6$ tiles. It is important to carefully choose an adequate number of sub-images so that the aspect ratio of the resulting sub-images is similar to the original image.

Irrespective of image resolution, the total number of sub-images derived from a given image is completely defined by the value assigned to the parameter $N$. If there are $T=500$ images in the dataset, then the total number of sub-images is calculated as $T_{N}=T \times N$. For different tessellation scenarios, the total number of sub-images is provided in Table 1 . It is evident that for a relatively small number of $N$, a substantial number of dataset samples is generated. These sub-images (dataset samples) may or may not contain pixels associated with fire.

Two examples of images and the results of an image tessellation scheme based on $N=20$ are presented in Figure 4, where sub-images containing fire are shown with a red overlay. For example, a $4000 \times 3000$ image is tessellated into 20 sub-images with a resolution of $800 \times 750$. A sub-image of this size is considered suitable to be used in the process of training a deep learning model. Once the results of the selected image tessellation scheme are obtained, it is necessary to assign a label (fire or not fire) to all individual sub-images.
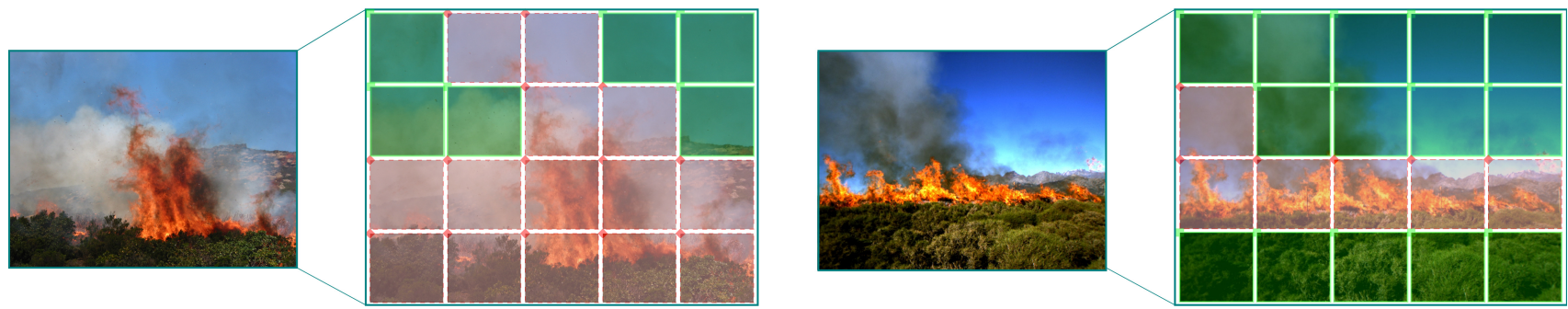

Figure 4. The process of image tessellation on two selected images from the CFDB dataset is illustrated. The configuration of $5 \times 4$ sub-images per image is shown. Labeling of sub-images is shown in different colors: (i) the image with a red diamond in the upper left corner represents a sub-image with fire, and (ii) the image with a green square in the upper left corner represents a sub-image without fire. The process results in a balanced number of fire and not-fire images.

\subsubsection{Formation of a Dataset for Deep Learning}

Manual classification of sub-images into two classes, fire and not fire, is a laborintensive and time-consuming task. It cannot be accomplished without professional knowledge and extensive experience in the field. Additionally, this approach does not scale very well for different tessellation strategies and large-scale datasets.

Labeling of sub-images is necessary to facilitate supervised learning. The process can be automated if the dataset is associated with ground truth images, or when an 
unsupervised method exists for the image classification. The CFDB dataset has associated ground truth images. Thus, we performed the same tessellation process on the ground truth images as on the original images, as was described in Section 3.2.1. The F1 score was calculated for each sub-image. We used an existing unsupervised method [19] to classify sub-images into two classes (fire and not-fire) based on the F1 score. We adapted this method to account for the specificities associated with the problem currently being addressed. Images that have a high F1 score are declared to belong to the "fire" class, while the rest belong to the "not-fire" class. In special cases, such as when a sub-image contains a very small number of fire pixels, we declare a sub-image to belong to the "not fire" class. This situation usually occurs on the edges of a sub-image that typically represents a small amount of smoke.

At the end of this step, a new dataset with only two classes is created. Additional steps are required to prepare the dataset for deep learning as is described in Section 4.1.

\subsection{LW-FIRE: A Lightweight CNN for Wildfire Image Classification}

In recent years, deep learning has demonstrated very promising results in a range of different engineering applications. DL-based models have the ability to automatically learn features from various data sources, and it has more robustness than other MLbased methods. Over the years, various DL-based models and applications have been developed [20-24]. However, one of the downsides of DL methods is an extensive training phase, which usually requires a large training dataset to attain the required functionality. More data always mean more training time and more computational resources-typically high-end GPUs or CPUs. Due to memory constraints, a model with a large number of learnable parameters and arithmetic operations is computationally expensive and can be trained for days to attain reasonable accuracy [25].

In this paper, we propose a new lightweight wildfire $\mathrm{CNN}$ for image classification. The CNN model can be trained in a reasonable time and computational resources (CPUs or GPUs, and memory), and is also suitable for real-time fire detection applications. LW-FIRE is an abbreviation for the Lightweight WildFIRE classification model. When a model has the real-time performance we add the "RT" prefix to the name of a model. The architecture of the basic Lightweight CNN for wildfire image classification is illustrated in Figure 5. The specification of layers and number of parameters is provided in Table 2. The structure of the $\mathrm{CNN}$ model was created with an extensive experimentation process and commonly observed practices from the DL community. From the experiments, we observed some favorable characteristics of the model on the validation dataset. Once the model achieved satisfied accuracy on the validation set, we tested our models on the three different testing sets. The process of evaluation is described in Section 5 .

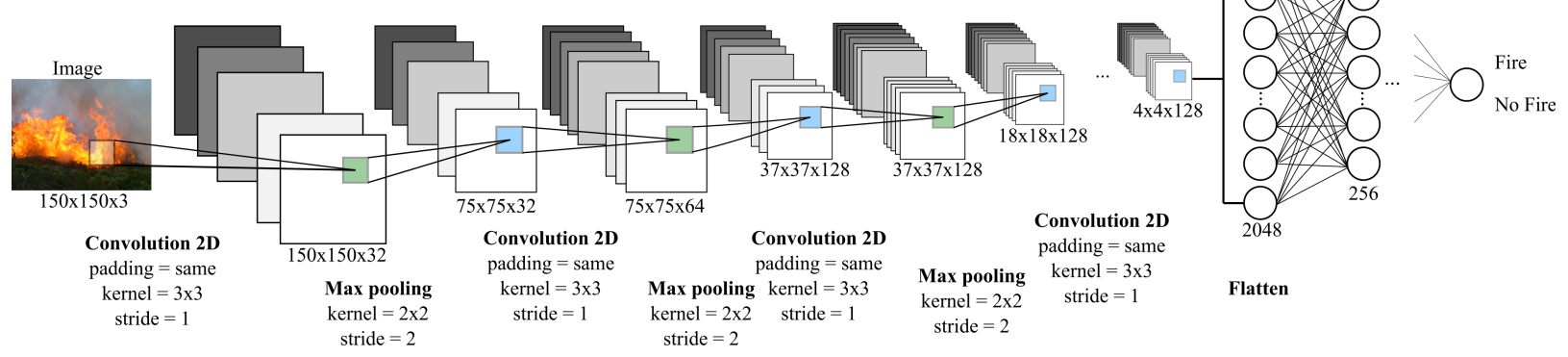

Figure 5. Illustrated architecture of LW-FIRE150 CNN model used for wildfire image classification. 
Table 2. Summary of the LW-FIRE150 deep convolutional neural network architecture.

\begin{tabular}{|c|c|c|c|c|}
\hline No. & Block Type & Block Description & Size of Feature Maps & No. Parameters \\
\hline 1 & Conv2D (ReLU) & 2D convolution layer & $(150,150,32)$ & 896 \\
\hline 2 & BatchNormalization & Batch normalization layer & $(75,75,32)$ & 128 \\
\hline 3 & MaxPooling2D & Max pooling for 2D data & $(75,75,32)$ & 0 \\
\hline 4 & Conv2D (ReLU) & 2D convolution layer & $(75,75,64)$ & 18,496 \\
\hline 5 & BatchNormalization & Batch normalization layer & $(75,75,64)$ & 256 \\
\hline 6 & MaxPooling2D & Max pooling for 2D data & $(37,37,64)$ & 0 \\
\hline 7 & Conv2D (ReLU) & 2D convolution layer & $(37,37,128)$ & 73,856 \\
\hline 8 & BatchNormalization & Batch normalization layer & $(37,37,128)$ & 512 \\
\hline 9 & MaxPooling2D & Max pooling for 2D data & $(18,18,128)$ & 0 \\
\hline 10 & Conv2D (ReLU) & 2D convolution layer & $(18,18,128)$ & 147,584 \\
\hline 11 & BatchNormalization & Batch normalization layer & $(18,18,128)$ & 512 \\
\hline 12 & MaxPooling2D & Max pooling for 2D data & $(9,9,128)$ & 0 \\
\hline 13 & Conv2D (ReLU) & 2D convolution layer & $(9,9,128)$ & 147,584 \\
\hline 14 & BatchNormalization & Batch normalization layer & $(9,9,128)$ & 512 \\
\hline 15 & Conv2D (ReLU) & 2D convolution layer & $(9,9,128)$ & 147,584 \\
\hline 16 & BatchNormalization & Batch normalization layer & $(9,9,128)$ & 512 \\
\hline 17 & MaxPooling2D & Max pooling for 2D data & $(4,4,128)$ & 0 \\
\hline 18 & Flatten & Flattens the input & 2048 & 0 \\
\hline 19 & Dense (ReLU) & Densely connected NN layer & 256 & 524,544 \\
\hline 20 & Dropout & Dropout regularization with rate 0.6 & 256 & 0 \\
\hline 21 & Dense (ReLU) & Densely connected NN layer & 128 & 32,896 \\
\hline 22 & Dropout & Dropout regularization with rate 0.3 & 128 & 0 \\
\hline 23 & Dense (ReLU) & Densely connected NN layer & 64 & 8256 \\
\hline 24 & Dropout & Dropout regularization with rate 0.15 & 64 & 0 \\
\hline 25 & Dense (ReLU) & Densely connected NN layer & 32 & 2080 \\
\hline 26 & Dropout & Dropout regularization with rate 0.075 & 32 & 0 \\
\hline 27 & Dense (ReLU) & Densely connected NN layer & 16 & 528 \\
\hline 28 & Dropout & Dropout regularization with rate 0.0325 & 16 & 0 \\
\hline \multirow[t]{2}{*}{29} & Dense (Sigmoid) & Densely connected NN layer & 1 & 17 \\
\hline & & & Total No. parameters: & $1,106,753$ \\
\hline
\end{tabular}

The input layer uses a set of 3-channel input images. The total number of images depends on the sub-image configuration chosen in the previous stage. We considered two configurations, as shown in Table 1: (i) $N=12$ and (ii) $N=42$, where the number of images $T_{N}=6000$ and $T_{N}=21,000$, respectively. The first convolution layer uses 32 output filters $(3 \times 3)$ followed by batch normalization and a max-pooling layer with $2 \times 2$ strides. The second convolution layer uses 64 output filters followed by batch normalization and max-pooling layer. Then, an additional convolution layer with 128 output filters is followed by batch normalization and max-pooling layers. Outputs from the last max-pooling layer are flattened and connected with several fully connected (FC or Dense) layers, where ReLU and Sigmoid activation functions are used, respectively. Non-linearity is introduced in all convolution layers with the ReLU activation function. The ReLU activation function 
significantly reduces the time for model convergence. The max-pooling layer is used after the convolution layers to reduce the spatial dimensionality of the output feature maps.

A batch normalization (BN) layer was added after every convolution layer to normalize the values in the batch of images and to encourage stable estimation. This technique reduces the overfitting problem [26]. Finally, we introduced several dense layers with dropout regularization techniques that randomly set hidden units to 0 with different frequency ratings. The dropout layer is applied only during the training stage.

\subsection{Inference}

After a DL-based model is trained and validated with the training and validation subsets to perform binary classification, its performance is evaluated on the test subset. The data split strategy is described in Section 4.1. The performance of the model is evaluated in terms of various measures based on the confusion matrix and processing speed defined in terms of average frames per second. Every test yields a prediction ("fire" or "not fire") and the time required for the prediction. We use this information to generate the testing accuracy of the model, execution time, and average frames per second.

\section{Results}

\subsection{Evaluation Criteria}

The transformed dataset consists of original images and sub-images, created in the preprocessing phase. The samples are split into three subsets: training, validation, and testing. We first randomly shuffled image data, and then divided them into $70 \%$ samples for training, $10 \%$ for validation, and $20 \%$ for testing. We trained our model on the training set and fine-tuned the hyperparameters on the validation set. The evaluation of the performance of the model was carried out on the testing subset. Table 3 provides more details about the number of samples used in each subset.

Table 3. Number of images in each sub-set of dataset used for training, validation and testing.

\begin{tabular}{cccc}
\hline $\mathbf{N}$ & Training Set (Fire, Not Fire) & Validation Set (Fire, Not Fire) & Test Set (Fire, Not Fire) \\
\hline 12 & 4552 & 652 & 1297 \\
& $(2735 ; 1817)$ & $(394 ; 258)$ & $(779 ; 518)$ \\
\hline 42 & 15,050 & 2149 & 4304 \\
& $(6208 ; 8842)$ & $(910 ; 1239)$ & $(1774 ; 2530)$ \\
\hline
\end{tabular}

The predictive ability of the models is evaluated using the evaluation criteria based on the confusion matrix (contingency table), with four different results: true positives (TP), true negatives (TN), false positives (FP), and false negatives (FN). Evaluation criteria used for the model performance are the sensitivity or true positive rate (TPR), specificity or true negative rate (TNR), fall-out or false positive rate (FPR), precision or positive predictive value (PPV), accuracy (ACC), F1 Score, Matthews correlation coefficient (MCC), and Fowlkes-Mallows index (FM), as is defined in Equations (1)-(8).

$$
\begin{gathered}
\mathrm{TPR}=\frac{\mathrm{TP}}{\mathrm{TP}+\mathrm{FN}} \\
\mathrm{FPR}=\frac{\mathrm{FP}}{\mathrm{FP}+\mathrm{TN}}=1-\mathrm{TNR} \\
\mathrm{TNR}=\frac{\mathrm{TN}}{\mathrm{TN}+\mathrm{TP}} \\
\mathrm{PPV}=\frac{\mathrm{TP}}{\mathrm{TP}+\mathrm{FP}} \\
\mathrm{ACC}=\frac{\mathrm{TP}+\mathrm{TN}}{\mathrm{TP}+\mathrm{TN}+\mathrm{FP}+\mathrm{FN}}
\end{gathered}
$$




$$
\begin{gathered}
\mathrm{F}_{1}=\frac{2 \cdot \mathrm{TP}}{2 \cdot \mathrm{TP}+\mathrm{FP}+\mathrm{FN}} \\
\mathrm{MCC}=\frac{\mathrm{TP} \times \mathrm{TN}-\mathrm{FP} \times \mathrm{FN}}{\sqrt{(\mathrm{TP}+\mathrm{FP})(\mathrm{TP}+\mathrm{FN})(\mathrm{TN}+\mathrm{FP})(\mathrm{TN}+\mathrm{FN})}} \\
\mathrm{FM}=\sqrt{\frac{\mathrm{TP}}{\mathrm{TP}+\mathrm{FP}} \times \frac{\mathrm{TP}}{\mathrm{TP}+\mathrm{FN}}}=\sqrt{\mathrm{PPV} \times \mathrm{TPR}}
\end{gathered}
$$

\subsection{Experimental Setup}

The model was designed with Keras with a TensorFlow backend and was trained and tested on an Intel Core i7-10700KF CPU @ 3.80GHz with 32GB memory.

An adaptive learning rate optimizer (Adam) was used as an optimization algorithm to adjust the weights of the CNN model. Adam is used for first-order gradient-based optimization of the stochastic objective function, which is based on adaptive estimates of lower-order moments [27]. We used a mini-batch training scheme for feeding image data into the proposed model. This scheme ensures that we trained the model with a balanced training set during the training epochs. Parameters used with the optimizer are the learning rate $\alpha=0.001$, the exponential decay rates for the first and second-moment estimates $\beta_{1}=0.9$ and $\beta_{2}=0.999$, and a very small number to prevent any division by zero $\epsilon=1 \times 10^{-8}$. The batch size is set to 32 for training and validation.

\subsection{Classification Performance of the Proposed Framework}

We consider two parameters when defining a model:

1. The type of image tessellation scheme, as defined in Table 3, and

2. The sample resolution $\mathrm{R}$ used for training, validation and testing, where $R=100,150$, 300 , and possible resolutions are defined as $100 \times 100,150 \times 150$, and $300 \times 300$.

Based on different combinations of two different image tessellation schemes and sample (sub-image) resolution, six different models are developed, as is shown in Table 4. In accordance with the associated image tessellation scheme, the performance of each model is presented in Table 5 .

Table 4. Six proposed models based on different image tessellation schemes and three different sub-image resolutions.

\begin{tabular}{clcc}
\hline No. & Model & Number of Tiles/Image & Resolution \\
\hline 1 & RT-LW-FIRE100 $_{12}$ & $N=12$ & $100 \times 100$ \\
\hline 2 & LW-FIRE150 $_{12}$ & $N=12$ & $150 \times 150$ \\
\hline 3 & LW-FIRE300 $_{12}$ & $N=12$ & $300 \times 300$ \\
\hline 4 & LW-FIRE100 $_{42}$ & $N=42$ & $100 \times 100$ \\
\hline 5 & LW-FIRE150 & & $150 \times 150$ \\
\hline 6 & LW-FIRE300 & & \\
\hline
\end{tabular}

The performance of each model is presented in Table 5. The resolution $R$ determines the total number of parameters. The models reach optimal performance in different epochs, as denoted in the column "Epochs". The maximum number of epochs used in all tests is 50 . More epochs were used in the experimental phase, but no significant improvement in terms of accuracy was observed; thus, we limited the number of epochs to 50 . The validation accuracy in Table 5 is the accuracy of the optimal model with respect to the specific epoch displayed in the column "Epochs". The average frames per second are calculated with respect to the number of images from the test set (Table 3).

The RT-LW-FIRE100 12 model has 877,377 parameters and requires approximately $50 \mathrm{~min}$ to train. Its validation accuracy is $97.8 \%$, and the testing accuracy is $96.1 \%$. The model can make a prediction in $32 \mathrm{~ms}$, and this enables processing of $31.25 \mathrm{FPS}$. In literature, the requirement 
for real-time capabilities is typically set to $30 \mathrm{fps}$, as is discussed in [28-30]. This sets the limitation for image processing time to at least $33.3 \mathrm{~ms}$. The reason for providing the real-time capabilities is to enable the implementation of our method on embedded devices, such as the ones used in drones. In return, this will help in alarming the firefighting forces immediately after the wildfire is detected.

The highest validation accuracy of the RT-LW-FIRE $100_{12}$ model is reached in 27 epochs. The other two models based on the same image tessellation scheme are denoted as LWFIRE150 12 and LWFIRE300 12 . These models reached the optimal validation accuracy after the 29th and 18th epochs, respectively.

The number of parameters increases with increasing image resolution. Even though more data is analyzed, results do not indicate a significant increase in performance, as is shown in the "Testing accuracy" column. However, the number of parameters causes the number of floating-point operations to increase. Hence, FPS is significantly decreased. Models also need more time to be trained, which is especially noticeable in the last configuration, where training can last up to seven hours.

Table 5. Different configurations and their performance measures for $N=12$ and $N=42$.

\begin{tabular}{|c|c|c|c|c|c|c|}
\hline Model & Image & No. Parameters & Epochs & $\begin{array}{l}\text { Validation } \\
\text { Accuracy }\end{array}$ & $\begin{array}{l}\text { Testing } \\
\text { Accuracy }\end{array}$ & FPS \\
\hline RT-LW-FIRE100 12 & $100 \times 100$ & 877,377 & $27 / 50$ & $97.8 \%$ & $96.1 \%$ & $31.25 \mathrm{fps}$ \\
\hline LW-FIRE150 12 & $150 \times 150$ & $1,106,753$ & $29 / 50$ & $98.13 \%$ & $95.83 \%$ & $27.7 \mathrm{fps}$ \\
\hline LW-FIRE $300_{12}$ & $300 \times 300$ & $3,236,673$ & $18 / 50$ & $97.5 \%$ & $95.4 \%$ & $19.6 \mathrm{fps}$ \\
\hline LW-FIRE100 42 & $100 \times 100$ & 877,377 & $32 / 50$ & $98.44 \%$ & $97.1 \%$ & $27 \mathrm{fps}$ \\
\hline LW-FIRE150 42 & $150 \times 150$ & $1,106,753$ & $35 / 50$ & $99.36 \%$ & $97.25 \%$ & $25.6 \mathrm{fps}$ \\
\hline LW-FIRE $300_{42}$ & $300 \times 300$ & $3,236,673$ & $6 / 50$ & $98.13 \%$ & $96.4 \%$ & $20 \mathrm{fps}$ \\
\hline
\end{tabular}

The LW-FIRE $100_{42}$ model has the same number of parameters as the RT-LW-FIRE100 42 . Its validation accuracy is $98.44 \%$, and its testing accuracy is $97.1 \%$. On the test dataset, it can make a prediction in $37 \mathrm{~ms}$, which is approximately $27 \mathrm{fps}$. This model is very close to reaching real-time capabilities. With better hardware or sophisticated optimizations (such as Intel's OpenVINO), this model could potentially be used in real-time applications. The best validation accuracy is reached after 32 epochs.

The next model, LW-FIRE150 42 , has 1,106,753 parameters. The best validation accuracy is reached after the 35th epochs of training and it is $99.36 \%$, while the testing accuracy is $97.25 \%$. An image is processed in $39 \mathrm{~ms}$, which implies that the model can predict 25.6 images (frames) per second. In terms of the performance on the test dataset, this model is the best model of all models that share this image tessellation scheme.

The model LW-FIRE $300_{42}$ has 3,236,673 parameters. The training time increased because the amount of data in the training phase also increased. The best validation accuracy is reached after the 6th epoch, and it is $98.1 \%$, while the testing accuracy is $96.4 \%$. On average, a test image is processed in $51 \mathrm{~ms}$, which indicates a processing speed of 19.6 frames per second.

In Figure 6, we present the overall performance of the best performing model (LWFIRE150 42 in terms of accuracy on the testing subset. The model displays stability and robustness. It also shows that adding the batch normalization and dropout layers prevented overfitting. 

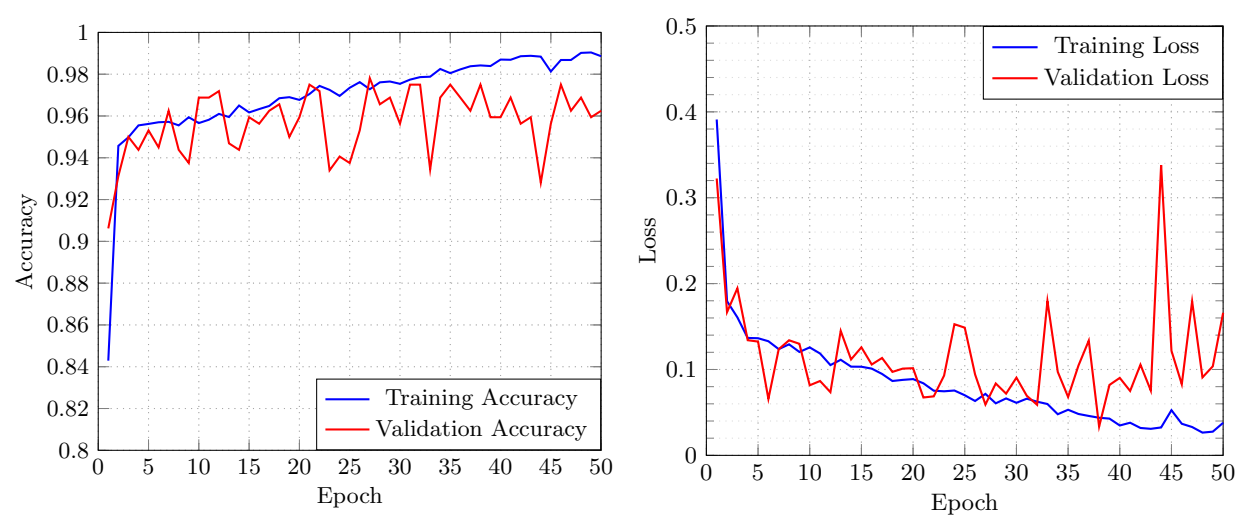

Figure 6. The overall performance of the LW-FIRE $150_{42}$ model with the highest accuracy on the testing set: training and validation accuracy (left) and loss (right).

\subsection{Multiclass Classification}

In this paper, we addressed the problem of wildfire image classification. We described a new deep learning-based model, and the dataset transformation on the example of two classes, namely "fire" and "not fire". In this subsection, we provide sufficient evidence that our solution can be extended to work on multiclass classification.

The number of possible classes is typically related to a specific application domain. Since we addressed the classification of wildfire images, we believe that the extension of this problem to include the class "smoke" is a logical choice. To prepare the dataset for multiclass classification, we use the same image tessellation technique described in Section 3.2.1, but now we also define a third class: "smoke". The sub-images created in the image tessellation step need to be labeled with appropriate labels. Sub-images that are labeled as "fire" in the binary classification problem can still be used. On the other hand, sub-images that are labeled as "not fire" can contain information that is associated with smoke or not smoke or fire. Thus, we manually selected sub-images associated with smoke from the "not fire" class, and labeled them as "smoke". The rest of the images remained with the label "not fire". In Figure 7, we provide a visual representation of how an image can be divided into three classes. Next, the labels are encoded as categorical vectors. The same technique that is described here can be applied to other application domains. Once the dataset is prepared, the CNN model needs to be modified to support the multiclass classification.

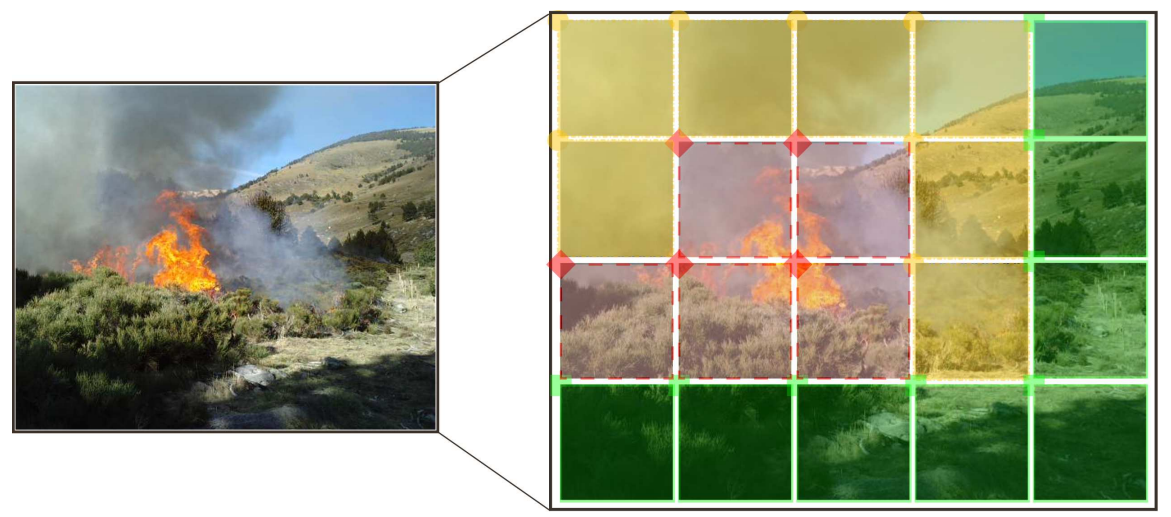

Figure 7. The process of image tessellation and labelling for multiclass classification is illustrated. Labeling of sub-images is shown in different colors in the upper left corner: (i) the image with a red diamond represents a sub-image with fire, (ii) the image with a green square represents a sub-image with no fire, and (iii) the image with a yellow circle represents a sub-image with smoke. (Best viewed in color.) 
Multiclass classification is demonstrated with the LW-FIRE150 42 model. The model is modified to generate three output signals instead of the one which is used for binary classification. During the training, the categorical cross-entropy function is used as a loss function. The same strategy is used to divide the samples, i.e., $70 \%$ of samples were used for training, $10 \%$ for validation, and $20 \%$ for testing. The model was trained for 50 epochs. The best validation accuracy was obtained after the 39th epoch, and the accuracy was 95.3\%. After testing our model on a testing subset, we noticed that the overall accuracy increased to $98.6 \%$. This is because the sub-images from the "not fire" class are divided into two classes, which increased the differentiation between the "not fire" and "smoke" classes, and, subsequently, the "fire" class. Hence, the model identified sub-images with fire more easily. Analysis of results revealed the increase in true positives and the decrease in false negatives, which had a positive effect on the majority of metrics used in this paper.

\section{Discussion}

In this section, we provide a comparison between the six state-of-the-art models discussed in Section 2 and the best models proposed in this work. We measured the performance of all models on the same hardware configuration and provide the results of eight metrics described in Section 4.1. We evaluated the following models:

1. FireNet [6];

2. InceptionNetV1-OnFire [6];

3. InceptionNetV3-OnFire [7];

4. InceptionNetV4-OnFire [7];

5. NasNet-A-OnFire [8];

6. ShuffleNetV2-OnFire [8];

7. Our LW-FIRE $100_{12}$ model, and;

8. Our LW-FIRE150 42 model.

Models 1-6 were trained on the dataset presented in [6]. The dataset was created by combining several other datasets, i.e., data from Chenebert et al. [10] (75,683 images), Steffens et al. [11] (20,593 images), also known as the "furg-fire-dataset" [12], and material from public video sources (youtube.com: 269,426 images). The authors then extracted 23,408 images from this collection to create a new dataset for training (12,550 images of fire, and 10,858 images of not-fire). The testing subset consists of 2931 images.

Models 7-8 were trained on the transformed CFDB dataset, as described in Section 3. The generalization of two of our models was demonstrated not only on the testing subset of this dataset but also on a testing subset from [6]. Besides the model's architecture, better generalization is achieved through the introduction of the dataset transformation technique, which increased the data usage from the dataset. To provide a fair comparison between the presented models, we performed two experiments:

1. Experiment I: All models have been tested on the Dunnings dataset used in [6]. Results are presented in Table 6;

2. Experiment II: All models have been testes on the dataset configurations created in this work based on the CFDB dataset. Results are presented in Tables 7 and 8 , for $N=12$ and $N=42$, respectively.

In Experiment I, we used a testing subset from [6], which consists of 2931 images extracted from six video sources. Some examples of images are provided in Figure 8, where we show the first and last frame of six video sources from the testing subset. In Table 6, we present the results. Models 1-6 have been evaluated on the original images, while our model LW-FIRE150 42 was evaluated on $2 \times 2$ sub-images. In this case, an image was considered to belong to the "fire" class if any of the sub-images has been classified as a "fire" image.

In Figure 8, we show the performance on $2 \times 2$ sub-images of two models: InceptionOne V4-OnFire, which has the best overall performance on this dataset, and our LW-FIRE $150_{42}$. The state-of-the-art models performed better on the original size images, 
while LW-FIRE150 42 performed better on sub-images, as was expected. We evaluated our model on $2 \times 2$ sub-images to show that our model can achieve a higher true positive rate (TPR) than the state-of-the-art models, while in other metrics, it shows comparable results. The results of our model could be improved if the false positive rate (FPR) is improved, which would have a positive effect on other metrics. Our model also outperformed FireNet, InceptionNetV1-OnFire, InceptionNetV3-OnFire, and ShuffleNetV2-OnFire when we consider metrics such as ACC, F1, MCC, and FM.

Table 6. Comparison with the state of the art on the dataset presented in [6]. Our model was not trained on this dataset, though other models have been trained and tested on this dataset. * Evaluated on $2 \times 2$ tiles of images in this dataset (as presented in Figure 8).

\begin{tabular}{clcccccccc}
\hline$\#$ & Model & TPR & FPR & TNR & PPV & ACC & F1 & MCC & FM \\
\hline$\# 1$ & FireNet [6] & 0.846 & 0.0831 & 0.916 & 0.910 & 0.881 & 0.877 & 0.765 & 0.878 \\
\hline$\# 2$ & InceptionNetV1-OnFire [6] & 0.810 & 0.0666 & 0.933 & 0.924 & 0.871 & 0.863 & 0.749 & 0.865 \\
\hline$\# 3$ & InceptionNetV3-OnFire [7] & 0.949 & 0.078 & 0.9218 & 0.9239 & 0.9358 & 0.9367 & 0.872 & 0.936 \\
\hline$\# 4$ & InceptionNetV4-OnFire [7] & 0.9481 & $\mathbf{0 . 0 4 7 7}$ & $\mathbf{0 . 9 5 2}$ & $\mathbf{0 . 9 5 2}$ & $\mathbf{0 . 9 5 0}$ & $\mathbf{0 . 9 5 0}$ & $\mathbf{0 . 9 0 0}$ & $\mathbf{0 . 9 5 0}$ \\
\hline$\# 5$ & NasNet-A-OnFire [8] & 0.973 & 0.082 & 0.917 & 0.922 & 0.945 & 0.947 & 0.892 & 0.947 \\
\hline$\# 6$ & ShuffleNetV2-OnFire [8] & 0.960 & 0.078 & 0.921 & 0.9247 & 0.941 & 0.942 & 0.883 & 0.942 \\
\hline$\# 8$ & Our LW-FIRE150 $_{42}{ }^{*}$ & $\mathbf{0 . 9 8 0}$ & 0.091 & 0.908 & 0.914 & 0.944 & 0.946 & 0.891 & 0.946 \\
\hline
\end{tabular}

Table 7. Comparison with the state of the art on the dataset presented in this paper $(N=12)$.

\begin{tabular}{|c|c|c|c|c|c|c|c|c|c|}
\hline$\#$ & Model & TPR & FPR & TNR & PPV & $\mathrm{ACC}$ & F1 & MCC & FM \\
\hline$\# 1$ & FireNet [6] & 0.727 & 0.0559 & 0.944 & 0.928 & 0.835 & 0.816 & 0.688 & 0.8226 \\
\hline \#2 & InceptionNetV1-OnFire [6] & 0.708 & 0.055 & 0.944 & 0.926 & 0.826 & 0.803 & 0.671 & 0.810 \\
\hline \#3 & InceptionNetV3-OnFire [7] & 0.794 & 0.028 & 0.971 & 0.964 & 0.882 & 0.871 & 0.777 & 0.875 \\
\hline$\# 4$ & InceptionNetV4-OnFire [7] & 0.869 & 0.036 & 0.963 & 0.959 & 0.916 & 0.912 & 0.836 & 0.913 \\
\hline$\# 5$ & NasNet-A-OnFire [8] & 0.820 & 0.0637 & 0.936 & 0.927 & 0.878 & 0.870 & 0.761 & 0.872 \\
\hline$\# 6$ & ShuffleNetV2-OnFire [8] & 0.820 & 0.025 & 0.974 & 0.970 & 0.897 & 0.889 & 0.804 & 0.892 \\
\hline \#7 & Our RT-LW-FIRE100 $_{12}$ & 0.966 & 0.063 & 0.936 & 0.938 & 0.951 & 0.952 & 0.903 & 0.952 \\
\hline
\end{tabular}

Table 8. Comparison with the state of the art on the dataset presented in this paper $(N=42)$.

\begin{tabular}{llcccccccc}
\hline$\#$ & Model & TPR & FPR & TNR & PPV & ACC & F1 & MCC & FM \\
\hline$\# 1$ & FireNet [6] & 0.695 & 0.039 & 0.960 & 0.946 & 0.827 & 0.801 & 0.679 & 0.810 \\
\hline$\# 2$ & InceptionNetV1-OnFire [6] & 0.598 & 0.020 & 0.979 & 0.966 & 0.788 & 0.738 & 0.624 & 0.760 \\
\hline$\# 3$ & InceptionNetV3-OnFire [7] & 0.769 & 0.030 & 0.969 & 0.961 & 0.869 & 0.854 & 0.753 & 0.860 \\
\hline$\# 4$ & InceptionNetV4-OnFire [7] & 0.753 & 0.0328 & 0.967 & 0.958 & 0.860 & 0.843 & 0.737 & 0.849 \\
\hline$\# 5$ & NasNet-A-OnFire [8] & 0.730 & $\mathbf{0 . 0 0 9}$ & $\mathbf{0 . 9 9 0}$ & $\mathbf{0 . 9 8 7}$ & 0.860 & 0.839 & 0.746 & 0.849 \\
\hline$\# 6$ & ShuffleNetV2-OnFire [8] & 0.791 & 0.013 & 0.986 & 0.983 & 0.889 & 0.877 & 0.793 & 0.882 \\
\hline$\# 8$ & Our LW-FIRE150 $_{42}$ & $\mathbf{0 . 9 7 6}$ & 0.030 & 0.969 & 0.970 & $\mathbf{0 . 9 7 3}$ & $\mathbf{0 . 9 7 3}$ & $\mathbf{0 . 9 4 6}$ & $\mathbf{0 . 9 7 3}$ \\
\hline
\end{tabular}

The classification accuracy is demonstrated in Figure 8. For example, InceptionOneV4OnFire (A) could not classify one of the sub-images as fire in the first frame of Video 1 and 2, while the last frame of Video 2 misclassified a fire truck as a "fire". In the first frame of Video 4, the model misclassified smoke as fire on one of the sub-images. In the last frame of Video 4, it failed to classify two sub-images as fire, and in Video 6, one sub-image as 
fire. In these examples, our model (B) correctly classified sub-images as "fire" or "not-fire" images. However, it misclassified sub-images in Video 3, where both algorithms have shown bad classification results. The results of the first frame of Video 3, 5, and 6, and the last frame of Video 1 and Video 5 show identical results of both models. On this statistical sample, LW-FIRE150 42 performed better, and it showed better performance in the overall results in this experiment, which is also reflected in Table 6.

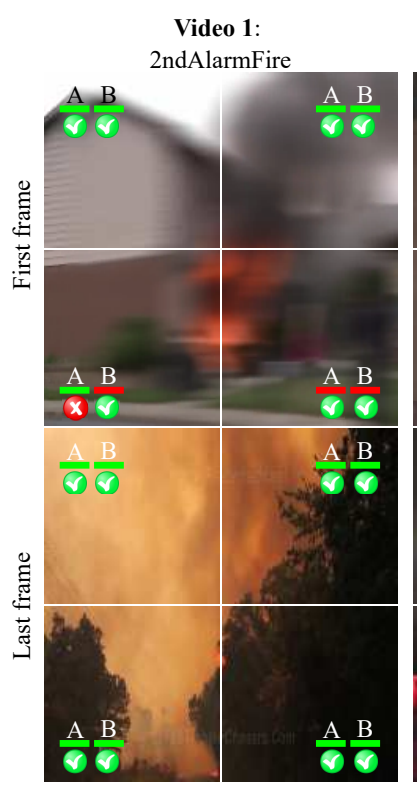

Video 4:
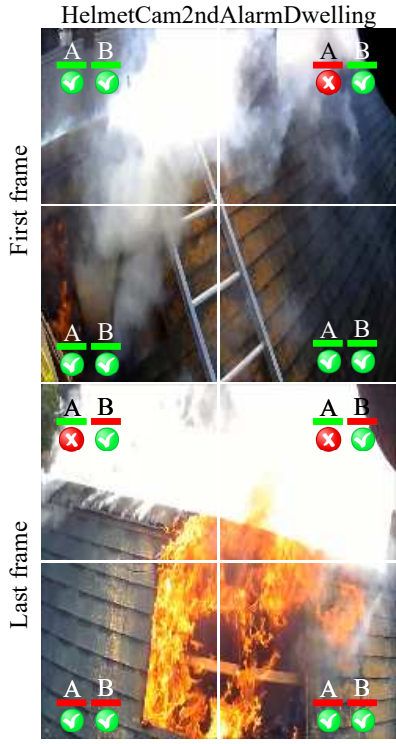

Video 2:

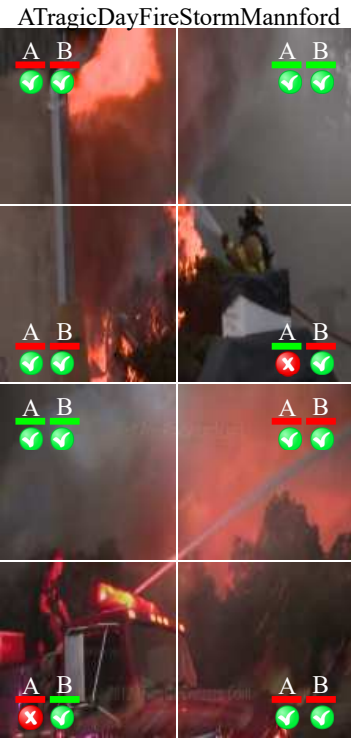

Video 5:

Ogdenhousefire
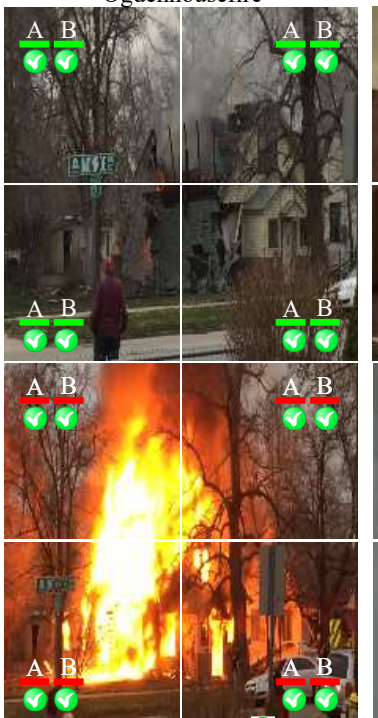

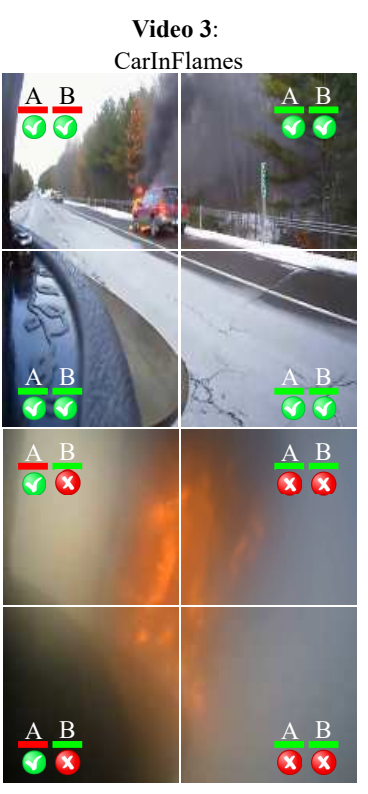

Video 6:

WaterMistFireDemonstration

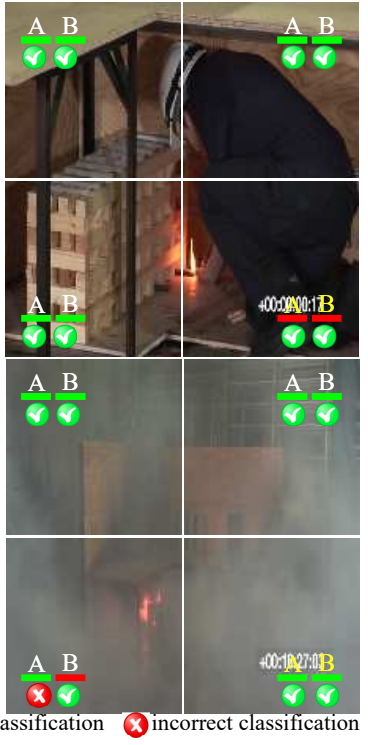

Figure 8. The performance of two models: (A) InceptionOneV4-OnFire, which has the best overall performance on this dataset out of the state of the art models, and (B) our LW-FIRE150 42 . We used the first and last frame from the six video sources from the dataset [6] to present various fire conditions. We tested models on $2 \times 2$ sub-images. The models' prediction results are indicated with green and red bars, where the green bar indicates that the fire was not detected, while the red bar indicates the presence of fire in the sub-image. Under each bar, we present the correct classification results. (Best viewed in color.)

In Experiment II, we tested all models on the dataset described in this paper, where $N=12$ and $N=42$. The testing subset for $N=12$ has 1297 images, while for $N=42$, it has 4304 images, as indicated in Table 3. The results are presented in Tables 7 and 8, 
for $N=12$ and $N=42$, respectively. The ShuffleNetV2-OnFire model has shown better performance in detecting frames that do not contain fire, reaching the FPR of 0.025 in case $N=12$. This is reflected in the results of TNR and PPV, which greatly rely on the FPR result. In the case of the NasNet-A-OnFire model, when $N=42$, FPR is 0.009 . This makes this model the best in terms of TNR and PPV. In terms of ACC, F1, MCC, and FM, our models showed better performance. In some cases, the performance is better than $10 \%$ when compared to the state-of-the-art models. This was not evident in Experiment I, where the results of our model were similar to the results of the state of the art. This proves that our model is a more robust solution for the problem of wildfire classification and detection.

In Table 9, we provide details about the number of parameters for each model. We also provide information about the real-time performance capabilities in terms of frames per second (FPS). The best performance is achieved with the ShuffleNetV2-OnFire model with 40 FPS. This model is also the smallest model in terms of parameters. Our model RTLW-FIRE100 12 achieves 31.25 FPS, and it is followed by LW-FIRE150 $0_{42}$ with 25.6 FPS. This is not a surprise since ShuffleNetV2 is a convolutional neural network that is optimized for a direct metric (speed) rather than an indirect metric like floating point operations [14]. It is built upon ShuffleNetV1 [31], which utilizes pointwise group convolutions, bottleneck-like structures, and a channel shuffle operation. In Table 6 , it can be observed that our model has a very similar performance as the ShuffleNetV2-OnFire in almost all categories, even though our model has never been trained on this dataset. In some categories, such as TPR, FPR, ACC, F1, MCC, and FM, it has even better performance. In Tables 7 and 8, the results for the transformed dataset are not very similar, and it can be observed that the LW-FIRE150 42 model outperforms the ShuffleNetV2-OnFire.

Table 9. Total number of parameters used in each model.

\begin{tabular}{clcc}
\hline$\#$ & Model & No. Parameters $\left(\times \mathbf{1 0}^{\mathbf{6}}\right)$ & FPS \\
\hline$\# 1$ & FireNet [6] & 6.79 & 17 \\
\hline$\# 2$ & InceptionNetV1-OnFire [6] & 1.2 & 8.4 \\
\hline$\# 3$ & InceptionNetV3-OnFire [7] & 0.96 & 12.1 \\
\hline$\# 4$ & InceptionNetV4-OnFire [7] & 7.18 & 15.4 \\
\hline$\# 5$ & NasNet-A-OnFire [8] & 3.2 & 7 \\
\hline$\# 6$ & ShuffleNetV2-OnFire [8] & 0.156 & 40 \\
\hline$\# 7$ & Our RT-LW-FIRE100 12 & 0.87 & 31.25 \\
\hline$\# 8$ & Our LW-FIRE15042 & 1.1 & 25.6 \\
\hline
\end{tabular}

After the analysis of the results, we can conclude that the methods presented in this paper show an important potential. The results can additionally be improved by increasing the size of the original dataset to include more sources of wildfire images and videos. Additionally, the performance could be improved by using special accelerators, such as advanced GPU cards.

\section{Conclusions}

In this paper, we propose a new method for transforming a dataset and training a custom-made lightweight deep convolutional neural network from scratch. With extensive experiments, we showed that this method can be successfully used to attain high performance on two datasets. To the best of our knowledge, this is the first time that such a data transformation model is introduced. We also show that our deep learning model can be successfully used to classify images on a different dataset that was not used in the training process. The method described here can be applied to other datasets in which the context of the image could not be lost in the tessellation process. 
A series of experimental results based on different configurations were carried out to identify the best model in terms of accuracy and timing performance. As a result, the models presented in this paper performed better when compared to the state of the art. The models were trained from scratch, and their performance results are better than the more complex deep convolutional models, which are based on models such as AlexNet and Google's Inception, or their transfer-learning variants.

Our model is suitable to be used in a lightweight UAV platform to monitor conditions on inaccessible lands for the purpose of reducing the risk of damaging wildfires.

\begin{abstract}
Author Contributions: Conceptualization, A.A. and E.B.; methodology, A.A.; software, A.A.; validation, A.A. and E.B.; formal analysis, A.A. and E.B.; investigation, E.B.; resources, A.A.; data curation, E.B.; writing—original draft preparation, A.A.; writing—review and editing, E.B.; visualization, A.A. All authors have read and agreed to the published version of the manuscript.
\end{abstract}

Funding: This research received no external funding.

Institutional Review Board Statement: Not applicable.

Informed Consent Statement: Not applicable.

Conflicts of Interest: The authors declare no conflict of interest.

\title{
References
}

1. National Fire News. Available online: https://www.nifc.gov/fire-information/nfn (accessed on 1 November 2021).

2. Wildland Fire Summary and Statistics Annual Report 2020. Available online: https://www.predictiveservices.nifc.gov/ intelligence/2020_statssumm/annual_report_2020.pdf (accessed on 1 November 2021).

3. Total Wildland Fires and Acres (1983-2020). Available online: https://www.nifc.gov/fire-information/statistics/wildfires (accessed on 1 November 2021).

4. MacDicken, K.G. Global forest resources assessment 2015: What, why and how? For. Ecol. Manag. 2015, 352, 3-8. [CrossRef]

5. Lyko, K.; Nitzschke, M.; Ngomo, A.C.N. Big data acquisition. In New Horizons for a Data-Driven Economy; Springer: Cham, Switzerland, 2016; pp. 39-61.

6. Dunnings, A.J.; Breckon, T.P. Experimentally defined convolutional neural network architecture variants for non-temporal real-time fire detection. In Proceedings of the 2018 25th IEEE International Conference on Image Processing (ICIP), Athens, Greece, 7-10 October 2018; pp. 1558-1562.

7. CA, G.S.; Bhowmik, N.; Breckon, T.P. Experimental exploration of compact convolutional neural network architectures for non-temporal real-time fire detection. In Proceedings of the 2019 18th IEEE International Conference On Machine Learning And Applications (ICMLA), Boca Raton, FL, USA, 16-19 December 2019; pp. 653-658.

8. Thomson, W.; Bhowmik, N.; Breckon, T.P. Efficient and Compact Convolutional Neural Network Architectures for Non-temporal Real-time Fire Detection. In Proceedings of the 2020 19th IEEE International Conference on Machine Learning and Applications (ICMLA), Miami, FL, USA, 14-17 December 2020; pp. 136-141.

9. Sousa, M.J.; Moutinho, A.; Almeida, M. Wildfire detection using transfer learning on augmented datasets. Expert Syst. Appl. 2020, 142, 112975. [CrossRef]

10. Chenebert, A.; Breckon, T.P.; Gaszczak, A. A non-temporal texture driven approach to real-time fire detection. In Proceedings of the 2011 18th IEEE International Conference on Image Processing, Brussels, Belgium, 11-14 September 2011; pp. 1741-1744.

11. Steffens, C.R.; Rodrigues, R.N.; da Costa Botelho, S.S. Non-stationary VFD evaluation kit: Dataset and metrics to fuel video-based fire detection development. In Robotics; Springer: Berlin/Heidelberg, Germany, 2016; pp. 135-151.

12. Furg-Fire-Dataset. Available online: https://github.com/steffensbola/furg-fire-dataset (accessed on 1 November 2021).

13. Zoph, B.; Vasudevan, V.; Shlens, J.; Le, Q.V. Learning transferable architectures for scalable image recognition. In Proceedings of the IEEE Conference on Computer Vision and Pattern Recognition, Salt Lake City, UT, USA, 18-23 June 2018; pp. 8697-8710.

14. Ma, N.; Zhang, X.; Zheng, H.T.; Sun, J. Shufflenet v2: Practical guidelines for efficient cnn architecture design. In Proceedings of the European Conference on Computer Vision (ECCV), Munich, Germany, 8-14 September 2018; pp. 116-131.

15. Jadon, A.; Omama, M.; Varshney, A.; Ansari, M.S.; Sharma, R. FireNet: A specialized lightweight fire \& smoke detection model for real-time IoT applications. arXiv 2019, arXiv:1905.11922.

16. FireNet Dataset. Available online: https://github.com/arpit-jadon/FireNet-LightWeight-Network-for-Fire-Detection (accessed on 1 November 2021).

17. Corsican Fire DataBase. Available online: http:/ / cfdb.univ-corse.fr/ (accessed on 1 November 2021).

18. Toulouse, T.; Rossi, L.; Campana, A.; Celik, T.; Akhloufi, M.A. Computer vision for wildfire research: An evolving image dataset for processing and analysis. Fire Saf. J. 2017, 92, 188-194. [CrossRef] 
19. Dzigal, D.; Akagic, A.; Buza, E.; Brdjanin, A.; Dardagan, N. Forest fire detection based on color spaces combination. In Proceedings of the 2019 11th International Conference on Electrical and Electronics Engineering (ELECO), Bursa, Turkey, 28-30 November 2019; pp. 595-599.

20. Deng, L.; Yu, D. Deep learning: Methods and applications. Found. Trends Signal Proc. 2014, 7, 197-387. [CrossRef]

21. Schmidhuber, J. Deep learning in neural networks: An overview. Neural Netw. 2015, 61, 85-117. [CrossRef] [PubMed]

22. Chen, X.W.; Lin, X. Big data deep learning: Challenges and perspectives. IEEE Access 2014, 2, 514-525. [CrossRef]

23. Yin, C.; Zhu, Y.; Fei, J.; He, X. A deep learning approach for intrusion detection using recurrent neural networks. IEEE Access 2017, 5, 21954-21961. [CrossRef]

24. Min, E.; Guo, X.; Liu, Q.; Zhang, G.; Cui, J.; Long, J. A survey of clustering with deep learning: From the perspective of network architecture. IEEE Access 2018, 6, 39501-39514. [CrossRef]

25. Shuvo, S.B.; Ali, S.N.; Swapnil, S.I.; Hasan, T.; Bhuiyan, M.I.H. A lightweight cnn model for detecting respiratory diseases from lung auscultation sounds using emd-cwt-based hybrid scalogram. IEEE J. Biomed. Health Informat. 2020, 25, 2595-2603. [CrossRef] [PubMed]

26. Goodfellow, I.; Bengio, Y.; Courville, A.; Bengio, Y. Deep Learning; MIT Press Cambridge: Cambridge, MA, USA, 2016 ; Volume 1.

27. Kingma, D.P.; Ba, J. Adam: A method for stochastic optimization. arXiv 2014, arXiv:1412.6980.

28. Redmon, J.; Divvala, S.; Girshick, R.; Farhadi, A. You only look once: Unified, real-time object detection. In Proceedings of the IEEE Conference on Computer Vision and Pattern Recognition, Las Vegas, NV, USA, 27-30 June 2016; pp. 779-788.

29. Hou, R.; Li, J.; Bhargava, A.; Raventos, A.; Guizilini, V.; Fang, C.; Lynch, J.; Gaidon, A. Real-time panoptic segmentation from dense detections. In Proceedings of the IEEE/CVF Conference on Computer Vision and Pattern Recognition, Seattle, WA, USA, 14-19 June 2020; pp. 8523-8532.

30. Wang, X.; Huang, Z.; Liao, B.; Huang, L.; Gong, Y.; Huang, C. Real-time and accurate object detection in compressed video by long short-term feature aggregation. Comput. Vis. Image Underst. 2021, 206, 103188. [CrossRef]

31. Zhang, X.; Zhou, X.; Lin, M.; Sun, J. Shufflenet: An extremely efficient convolutional neural network for mobile devices. In Proceedings of the IEEE Conference on Computer Vision and Pattern Recognition, Salt Lake City, UT, USA, 18-22 June 2018; pp. 6848-6856. 